\title{
Alkali metal cations can inhibit non-covalent catalysis
}

\author{
Nazar Rad ${ }^{*, \dagger}$ and Volodymyr Sashuk $*, \dagger$ \\ $\dagger$ Institute of Physical Chemistry, Polish Academy of Sciences, Kasprzaka 44/52, 01-224 \\ Warsaw, Poland
}

The study concerns the effect of inorganic salts on supramolecular catalysis. The model reaction is the acid hydrolysis of the ammonium phenyl acetate derivative promoted by cucurbit[7]uril macrocycle. When salt is absent, the macrocycle is insensitive to the ionic strength of the solution, and the reaction rate linearly depends on the concentration of hydronium ions $\left(\mathrm{H}_{3} \mathrm{O}^{+}\right)$. After the addition of inorganic salts, in particular, $\mathrm{Na}^{+}$and $\mathrm{K}^{+}$ions, the catalytic effect of the macrocycle is suppressed. The kinetic and binding data collected by us evidence the formation of the ternary complexes between the cations, macrocycle, and substrate, which are less prone to $\mathrm{H}_{3} \mathrm{O}^{+}$attack. This type of inhibition corresponds to a rare uncompetitive model in contrast to a more common competitive one that relies on the displacement of the substrate. This study shows that special care must be taken when studying catalysis in solutions that contain metal cations, such as regular water and inorganic buffers.

Keywords: cucurbituril, acid hydrolysis, phenyl acetate, sodium, potassium, salt effect, ternary complex, uncompetitive inhibition.

\section{Introduction}

Monovalent $\mathrm{Na}^{+}$and $\mathrm{K}^{+}$ions play critical roles in proper cell function and regulation. The well-known $\mathrm{Na} / \mathrm{K}$ pump creates ionic gradients controlling cellular volume and ensuring the transduction of nerve signals, the transportation of nutrients, acid-base homeostasis, to name a few. On the other hand, the same ions are the main components of buffer solutions widely used by researchers to maintain a constant $\mathrm{pH}$, particularly when studying catalysis. There was a general opinion that inorganic buffers are innocent mediums until the recent report of Gibbs and co-workers ${ }^{1}$, who revealed their detrimental influence on host-guest complexation that resembles the formation of the enzyme-substrate complex and always precedes catalysis. Despite ample evidence of cation binding to enzyme mimics ${ }^{2-6}$, their potential impact on catalysis continues to be unappreciated. To date, attention has been rather paid to the positive effect of inorganic cations. For example, sodium ions by the complexation with crown ether-based enzyme mimics were shown to promote condensation reaction ${ }^{7}$ and facilitate the release of a rhodium catalyst to enable asymmetric dehydrogenation ${ }^{8}$. Similarly, potassium ions combined with crown ether pockets were used to accelerate ester hydrolysis 9 . Besides, more heavy cations by coordinating to cucurbituril-based mimics were demonstrated to accelerate desillylation ${ }^{10}\left(\mathrm{Ag}^{+}\right)$, increase the chemoselectivity of deazotation ${ }^{11}\left(\mathrm{Ag}^{+}\right)$, and the enantioselectivity of Diels-Alder reaction ${ }^{12}\left(\mathrm{Cu}^{2+}\right)$. Herein we show that the effect of cations can be the opposite, especially when working with water environments, which are often abundant in alkali metal ions.

\section{Results and discussion}

In search of a model system, we focused on cucurbituril (CB) macrocycles, which due to the presence of multiple negative dipoles on their rims, establish strong electrostatic attractions with cations. Particularly, their ability to interact with hydronium ions $\left(\mathrm{H}_{3} \mathrm{O}^{+}\right)$is widely utilized in acid catalysis. Cucurbiturils are known to promote the hydrolytic cleavage of amides ${ }^{13}$, benzoyl chlorides ${ }^{14}$, esters ${ }^{15}$, ethers ${ }^{16}$, triazenes $^{17}$, oximes ${ }^{13}$, Schiff bases ${ }^{18}$, as 
well as the formation of hydrazones ${ }^{19}$. The activity of these versatile enzyme mimics strongly depends on $\mathrm{pH}$. Generally, the lower the $\mathrm{pH}$, the lower their efficiency ${ }^{13}$. Accordingly, the same effect should be expected for metal cations; however, it is obscured by hydronium ions, the concentration of which is typically regulated by inorganic buffers.

To separate the effect of hydronium ions from other ions, we first buffered solutions with neat acids, and then we added salts. Ester hydrolysis was chosen as a model reaction. This reaction is slow enough for observation at low $\mathrm{pH}$. Moreover, the irreversibility of the ester hydrolysis in an aqueous solution facilitates data interpretation. As a catalyst, we chose a water-soluble cucurbit[7]uril (CB7). The effectiveness of CB7 depends on the concentration of a substrate-catalyst complex. To increase the affinity of $\mathrm{CB} 7$, we have used cationic parasubstituted ammonium phenyl acetate substrate (PhAc). Besides, the rate-determining step of the hydrolysis is the protonation of the ester group ${ }^{20}$. Thus, we expected the stabilization of a positively charged transition state by CB7 rims and the promotion of the ester hydrolysis (Fig 1a).

The binding constant of PhAc@CB7 complex determined by ${ }^{1} \mathrm{H}$ NMR titration is equal to $(1.5 \pm 0.42) \cdot 10^{5} M^{-1}$. The addition of CB7 to PhAc shifts all proton signals upfield (ESI, Fig. S1). The most shifted signal belongs to aromatic proton $3 c$ in meta-position to acetate group. Significantly shifted are also the proton resonances of ammonium group $5 c$. The less shifted signal corresponds to the proton of acetate group $1 c$. Based on this, we can conclude that the phenyl ring and ammonium group are buried deep inside the cavity, and the acetate group is localized close to one of the CB7 rims.

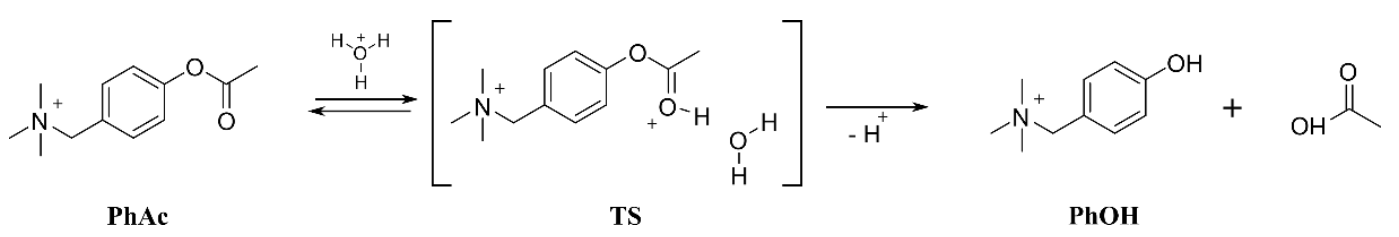

Scheme 1. Acid hydrolysis of PhAc.

In the salt-free solution, the hydrolysis rate of $\mathrm{PhAc} k_{f}$ (the reaction is depicted in Scheme 1) depends linearly on the concentration of $\mathrm{H}_{3} \mathrm{O}^{+}$ions in the range of $\mathrm{pH}$ between 0.75 to 2.5 (Fig. 1b). Since both $\mathrm{PhAc}$ and $\mathrm{H}_{3} \mathrm{O}^{+}$involved in the formation of the transition state are positively charged species, $k_{f}$ depends also on the ionic strength of the solution (eq 1). That is, a typical positive primary salt effect ${ }^{21}$ is observed.

$$
k_{f}=k_{f}^{0} *\left[D^{+}\right] * 10^{-2 *\left(-\frac{0.509 * \sqrt{I}}{1+\sqrt{I}}+0.14 * I\right)}
$$

where $k_{f}^{0}$ stands for the rate constant, $\left[D^{+}\right]$denotes the concentration of deuterium ion, $I$ is the ionic strength.

Adding $1.1 \mathrm{eq}$ of $\mathrm{CB} 7$ to PhAc accelerates the reaction by more than two orders of magnitude. In this case, the rate of hydrolysis $k_{b}$ also increases linearly as $\mathrm{pH}$ decreases (Fig $1 b$ ), although it is insensitive to the ionic strength (eq 2).

where $k_{b}^{0}$ is the rate constant.

$$
k_{b}=k_{b}^{0} *\left[D^{+}\right]
$$

The lack of the primary salt effect for encapsulated $\mathrm{PhAc}$ is explained by the presence of the hydrophobic cavity that isolates the reaction center (acetate group) from the charged anchor (ammonium group).

$$
\alpha=\frac{k_{b}}{k_{f}}
$$


Consequently, the acceleration factor $\alpha$, calculated as the ratio of the hydrolysis rate constants of the encapsulated and free $\mathrm{PhAc}$ (eq. 3) decreases as the ionic strength of the solution increases. That is, if the substrate is charged, the ionic-strength-independent acceleration factor $\alpha^{0}$ should be given instead. For CB7 promoted PhAc acid hydrolysis, $\alpha^{0}$ is equal to $263 \pm 12$.

(a)

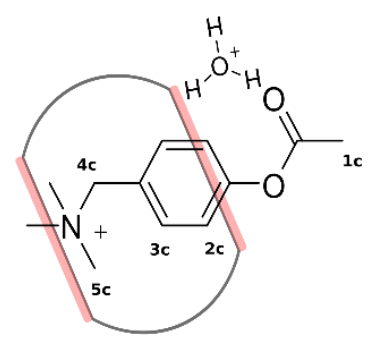

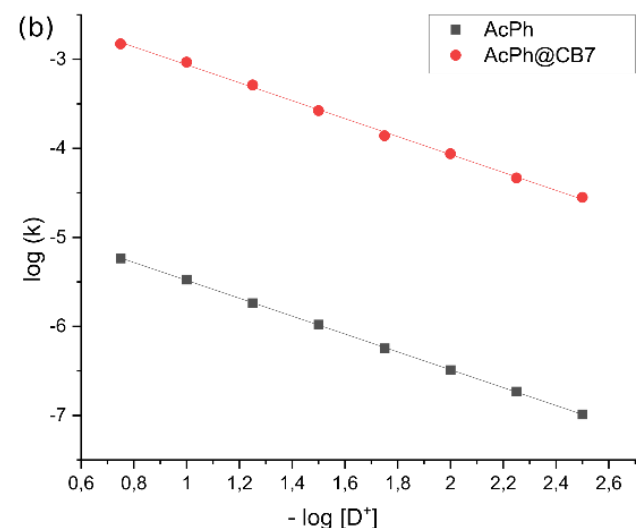

Figure 1. (a) Schematic depiction of PhAc@CB7 complex; (b) pD-profiles of PhAc and PhAc@CB7 hydrolysis.

In the next step, we studied the effect of sodium chloride. As expected, the addition of sodium chloride speeded up the hydrolysis of free PhAc due to the increase of ionic strength. Surprisingly, it also reduced the activity of CB7. The obtained kinetic data do not agree with competitive inhibition, where sodium cation competes with the substrate for the macrocycle (ESI, eq18). Moreover, the competitive inhibition scenario does not explain shifts in ${ }^{1} \mathrm{H}$ NMR spectra. Upon adding salt, the signal of $2 \mathrm{c}$ proton moves upfield, which can only be accounted for by the translocation of phenyl ring deeper into the CB7 cavity (Fig 2).
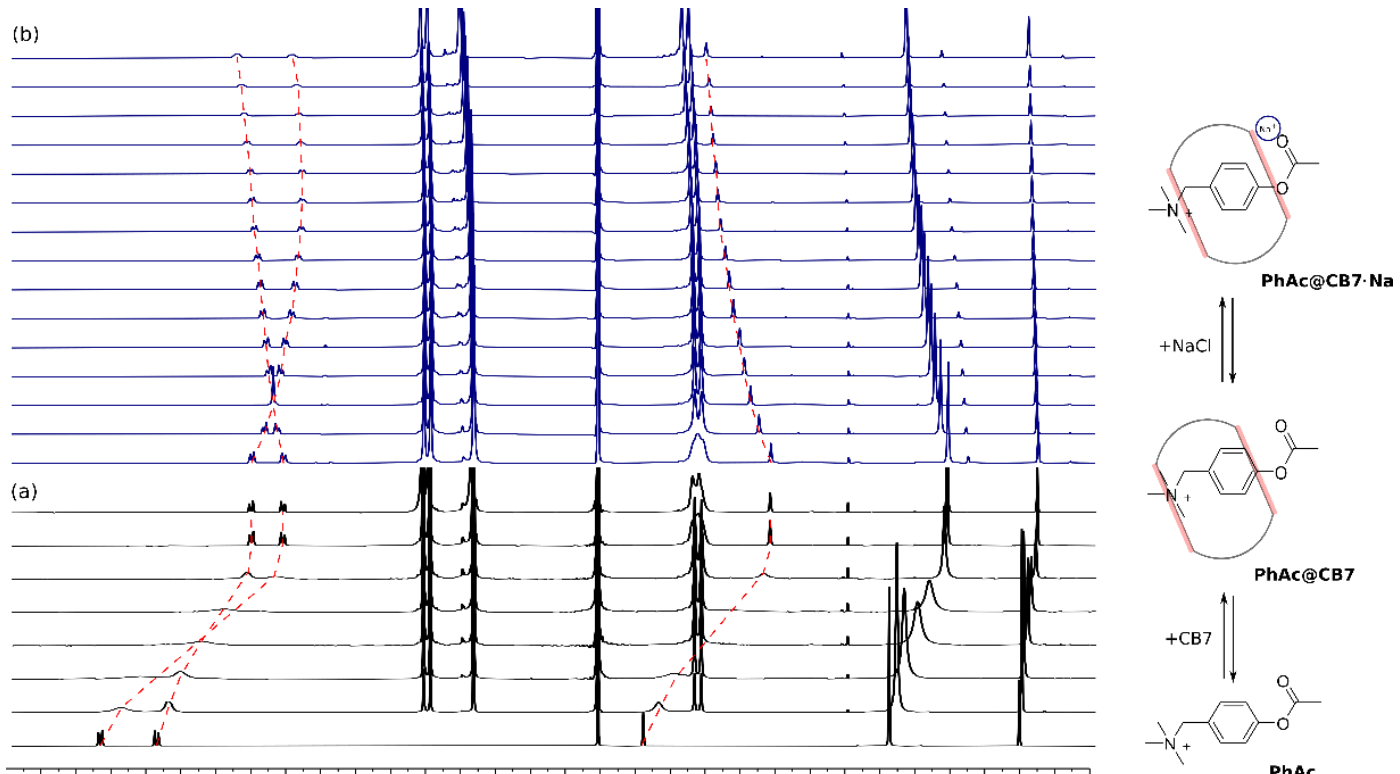

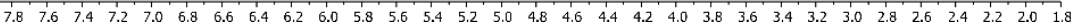

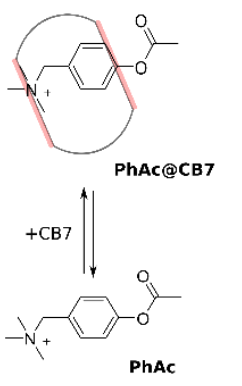

Figure 2. NMR titration experiments of PhAc with CB7 (spectra are shown in black) and PhAc@CB7 complex with $\mathrm{NaCl}$ (spectra are denoted in blue).

To elucidate the source of inhibition, we recorded NMR spectra in the presence of different salts. The screening has shown that the shielding of $2 c$ proton signals depends strongly on the nature of the cation and does not change when varying anion (ESI, Fig. S8). 
This observation is supported by acceleration factors, which are different for four cations, and are of practically the same value for four anions (ESI, table T2).

Thus, we consider the model of uncompetitive inhibition of CB7 by cation. According to this model, the cation binds to the CB7 portal next to the reactive center to afford a PhAc@CB7·Na ternary complex. The positively charged alkali cation destabilizes the conjugated acid of ester to slow down the acid hydrolysis. The formation of the ternary complex is supported by the mass analysis of the isolated PhAc@CB7 complex revealing a monosodium doubly charged molecular ion (ESI, Fig. S2).

The kinetic data of PhAc@CB7 hydrolysis in the presence of the different amounts of sodium chloride were employed to estimate the PhAc@CB7 affinity for sodium cation according to the model of uncompetitive inhibition. The obtained binding constant is almost identical to the constant determined by NMR titration within the statistical error. Thus, the deactivation of $\mathrm{CB} 7$ correlates with the cation-induced dislocation of the macrocycle toward the ester group.

The binding constants calculated for other cations also yield very close values for both methods. The cation affinity for PhAc@CB7 complex decreases in the same order as the inhibition efficiency of cations: $\mathrm{Na}^{+}>\mathrm{K}^{+}>\mathrm{Cs}^{+}>\mathrm{Li}^{+}$(Table T1). Therefore, the intermolecular gap between the CB7 rim and the ether group is in the range of 1-1.4 $\AA$, which corresponds to the most effective $\mathrm{Na}^{+}$and $\mathrm{K}^{+}$cations (Fig 3b).

Table 1. The binding constants of alkali cations with PhAc@CB7.

\begin{tabular}{|c|c|c|c|}
\hline & PhAc@CB7, kinetics & PhAc@CB7, NMR shifts & PhOH@CB7, NMR shifts \\
\hline $\mathrm{Li}^{+}$ & $1.8(0.7 \%)$ & $1.1(6.8 \%)$ & $0.9(4.5 \%)$ \\
\hline $\mathrm{Na}^{+}$ & $45.5(2.0 \%)$ & $46.1(4.8 \%)$ & $19.4(3.6 \%)$ \\
\hline $\mathrm{K}^{+}$ & $42.4(2.1 \%)$ & $43.3(6.7 \%)$ & $29.7(8.8 \%)$ \\
\hline $\mathrm{Cs}^{+}$ & $11.9(2.3 \%)$ & $10.8(4.8 \%)$ & $22.4(11.5 \%)$ \\
\hline
\end{tabular}

The numbers in parentheses show the relative mean deviation of the calculated data from the experimental ones.

The PhAc@CB7 complex binds sodium two times stronger than pure CB7 (K=21 \pm 2 $\left.\mathrm{M}^{-1}\right){ }^{22}$ This indicates that the organic guest reinforces the binding. The replacement of substrate $\mathrm{PhAc}$ by the product $\mathrm{PhOH}$ changes the affinity of the $\mathrm{CB} 7$-guest complex for cations. This is well seen on the ${ }^{1} \mathrm{H}$ NMR spectra of hydrolysis that signals positions of product PhOH@CB7 depend on the concentration of cations (ESI Fig. . The determined binding constants of PhOH@CB7 with cations decrease in the order $\mathrm{K}^{+}>\mathrm{Cs}^{+}>\mathrm{Na}^{+}>\mathrm{Li}^{+}$ (Fig 3b).
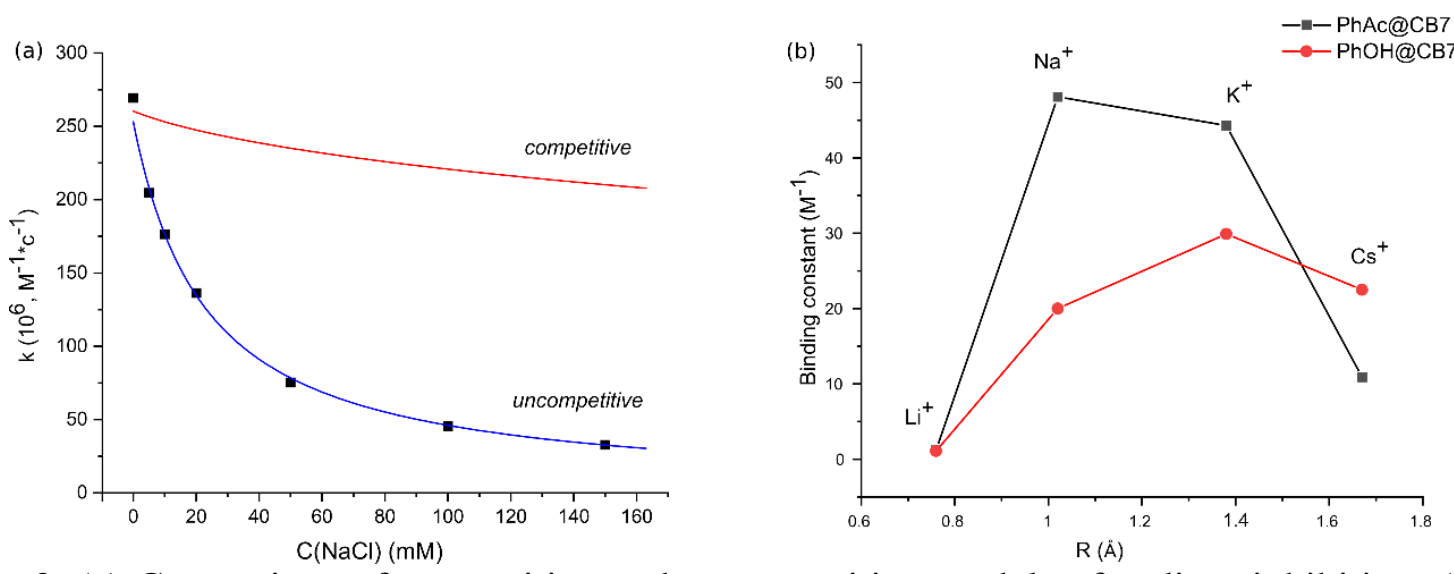

Figure 3. (a) Comparison of competitive and uncompetitive models of sodium inhibition; (b) Plots of binding constants vs. cation radius for substrate $\mathrm{PhAc}$ and product $\mathrm{PhOH}$. 
To summarize, we scrutinized the effect of ionic strength on supramolecular catalysis by cucurbit[7]uril macrocycle. The studied reaction was the acid hydrolysis of cationic phenyl acetate derivative. In the absence of inorganic salts, the macrocycle isolates the reaction center from the cationic anchor making the substrate and the macrocycle insensitive to the ionic strength of the solution. However, after the addition of salts, the catalytic activity of the macrocycle is suppressed. The reason for this is the formation of a ternary complex with alkali metal cations that is less susceptible to hydronium ion attack. This type of inhibition can be described by the uncompetitive model in which the substrate remains bound to the macrocycle instead of being displaced by the inhibitor. This work should change our view of inorganic buffers as innocent reaction mediums. Moreover, the cation selectivity observed during the formation of ternary complexes can be the basis for the development of new cation receptors.

\section{ASSOCIATED CONTENT}

Supporting Information. Synthetic procedures, characterization, binding and kinetic studies.

\section{AUTHOR INFORMATION}

Corresponding Author

*E-mail: nrad@ichf.edu.pl, vsashuk@ichf.edu.pl

\section{Author Contributions}

The manuscript was written through contributions of all authors.

All authors have given approval to the final version of the manuscript.

\section{Conflicts of interest}

There are no conflicts of interest to declare.

\section{Acknowledgements}

This work was financed by the National Science Centre of Poland (grant MINIATURA 3 no 2019/03/X/ST4/01017)

\section{References}

1. Jordan, J. H.; Ashbaugh, H. S.; Mague, J. T.; Gibb, B. C., Buffer and Salt Effects in Aqueous Host-Guest Systems: Screening, Competitive Binding, or Both? J Am Chem Soc 2021, 143 (44), 1860518616.

2. Marquez, C.; Hudgins, R. R.; Nau, W. M., Mechanism of host-guest complexation by cucurbituril. J Am Chem Soc 2004, 126 (18), 5806-5816.

3. Miskolczy, Z.; Megyesi, M.; Biczok, L.; Prabodh, A.; Biedermann, F., Kinetics and Mechanism of Cation-Induced Guest Release from Cucurbit[7]uril. Chem-Eur J 2020, 26 (33), 7433-7441.

4. Yin, H.; Rosas, R.; Gigmes, D.; Ouar, O.; Wang, R. B.; Kermagoret, A.; Bardelang, D., Metal Actuated Ring Translocation Switches in Water. Org Lett 2018, 20 (11), 3187-3191.

5. Megyesi, M.; Biczok, L.; Jablonkai, I., Highly sensitive fluorescence response to inclusion complex formation of berberine alkaloid with cucurbit[7] uril. J Phys Chem C 2008, 112 (9), 34103416. 
6. Thomas, S. S.; Tang, H.; Bohne, C., Noninnocent Role of $\mathrm{Na}+$ lons in the Binding of the NPhenyl-2-naphthylammonium Cation as a Ditopic Guest with Cucurbit[7]uril. J Am Chem Soc 2019, 141 (24), 9645-9654.

7. Lee, Y. J.; Liu, K. S.; Lai, C. C.; Liu, Y. H.; Peng, S. M.; Cheng, R. P.; Chiu, S. H., Na+ lons Induce the Pirouetting Motion and Catalytic Activity of [2]Rotaxanes. Chem-Eur J 2017, 23 (41), 97569760.

8. Ouyang, G. H.; He, Y. M.; Li, Y.; Xiang, J. F.; Fan, Q. H., Cation-Triggered Switchable Asymmetric Catalysis with Chiral Aza-CrownPhos. Angew Chem Int Edit 2015, 54 (14), 4334-4337.

9. Tozawa, T.; Tokita, S.; Kubo, Y., An allosteric crown ether-induced activity control for the cleavage of a phosphodiester bond. Tetrahedron Lett 2002, 43 (19), 3455-3457.

10. Lu, X. Y.; Masson, E., Silver-Promoted Desilylation Catalyzed by Ortho- and Allosteric Cucurbiturils. Org Lett 2010, 12 (10), 2310-2313.

11. Koner, A. L.; Marquez, C.; Dickman, M. H.; Nau, W. M., Transition-Metal-Promoted Chemoselective Photoreactions at the Cucurbituril Rim. Angew Chem Int Edit 2011, 50 (2), 545-548.

12. Zheng, L. F.; Sonzini, S.; Ambarwati, M.; Rosta, E.; Scherman, O. A.; Herrmann, A., Turning Cucurbit[8]uril into a Supramolecular Nanoreactor for Asymmetric Catalysis. Angew Chem Int Edit 2015, 54 (44), 13007-13011.

13. Klock, C.; Dsouza, R. N.; Nau, W. M., Cucurbituril-Mediated Supramolecular Acid Catalysis. Org Lett 2009, 11 (12), 2595-2598.

14. Basilio, N.; Garcia-Rio, L.; Moreira, J. A.; Pessego, M., Supramolecular Catalysis by Cucurbit[7]uril and Cyclodextrins: Similarity and Differences. J Org Chem 2010, 75 (3), 848-855. 15. Fierro, A.; Garcia-Rio, L.; Arancibia-Opazo, S.; Alcazar, J. J.; Santos, J. G.; Aliaga, M. E., Cucurbit[7]uril as a Supramolecular Catalyst in Base-Catalyzed Reactions. Experimental and Theoretical Studies on Carbonate and Thiocarbonate Hydrolysis Reactions. J Org Chem 2021, 86 (2), 2023-2027.

16. Scorsin, L.; Roehrs, J. A.; Campedelli, R. R.; Caramori, G. F.; Ortolan, A. O.; Parreira, R. L. T.; Fiedler, H. D.; Acuna, A.; Garcia-Rio, L.; Nome, F., Cucurbituril-Mediated Catalytic Hydrolysis: A Kinetic and Computational Study with Neutral and Cationic Dioxolanes in CB7. Acs Catal 2018, 8 (12), 12067-12079.

17. Sashuk, V.; Butkiewicz, H.; Fialkowski, M.; Danylyuk, O., Triggering autocatalytic reaction by host-guest interactions. Chem Commun 2016, 52 (22), 4191-4194.

18. Alcazar, J. J.; Geue, N.; Valladares, V.; Canete, A.; Perez, E. G.; Garcia-Rio, L.; Santos, J. G.; Aliaga, M. E., Supramolecular Control of Reactivity toward Hydrolysis of 7-Diethylaminocoumarin Schiff Bases by Cucurbit[7]uril Encapsulation. Acs Omega 2021, 6 (15), 10333-10342.

19. Rad, N.; Danylyuk, O.; Sashuk, V., Reversing Chemoselectivity: Simultaneous Positive and Negative Catalysis by Chemically Equivalent Rims of a Cucurbit[7]uril Host. Angew Chem Int Edit 2019, 58 (33), 11340-11343.

20. Said, Z.; Tillett, J. G., Acid-Catalyzed Hydrolysis of Phenyl Acetate. J Org Chem 1981, 46 (12), 2586-2588.

21. Bell, R. P.; Robson, M., Kinetic Salt Effects in Hydrolysis of Acetylcholine. T Faraday Soc 1964, 60 (4975), 893-\&.

22. Tang, H.; Fuentealba, D.; Ko, Y. H.; Selvapalam, N.; Kim, K.; Bohne, C., Guest Binding Dynamics with Cucurbit[7]uril in the Presence of Cations. J Am Chem Soc 2011, 133 (50), 2062320633. 